\title{
Análisis del costo del ausentismo laboral en personal de enfermería
}

\section{Analysis of the cost of work absenteeism in nursing personnel}

\author{
Paulina Tipantuña Malte ${ }^{1}$, Armando Sánchez Macías², Sofía Cheverria Rivera ${ }^{3}$, Rosa Andrade \\ Cepeda $^{4}$
}

\section{RESUMEN}

Objetivo: analizar el costo del ausentismo laboral en personal de enfermería de un Hospital público de la ciudad de San Luis Potosí, en México. Metodología: investigación cuantitativo retrospectiva a través de los registros de asistencia del año 2019, se identificaron 508 personas con ausentismo. El costo se determinó en base al salario diario por turno, estimado para tres categorías, se presentaron 3965 ausencias de las cuales $68 \%$ pertenecen a faltas injustificadas, y $31 \%$ a incapacidad médica. Resultados: el costo anual estimado fue de $\$ 6,676,134.62$ MXN. Los grupos que más ausencias presentaron y por ende más costos generaron fueron los correspondientes al mes de octubre, la categoría de enfermera profesional, el turno nocturno, en relación a incapacidad médica el mes de enero, la falta injustificada presentó el pico más alto en el mes de octubre. Conclusión: el ausentismo produce altos costos económicos, los cuales sobresalen en determinados grupos, al identificarlos se puede llevar un control de estos, y desarrollar estrategias que intervengan en las causas que lo originan, y así tomar decisiones oportunas y pertinentes.

Palabras clave: ausentismo laboral; investigación en administración de Enfermería; Personal de Enfermería; costos y análisis de costo.

\begin{abstract}
Objective: to analyze the cost of work absenteeism in nursing personnel of a public Hospital in the city of San Luis Potosí, in Mexico. Methodology: retrospective quantitative research through the attendance records of the year 2019, 508 people with absenteeism were identified. The cost was determined based on the daily salary per shift, estimated for three categories, there were 3,965 absences of which $68 \%$ correspond to unexcused absences and $31 \%$ to medical disability. Results: the estimated annual cost was $\$ 6,676,134.62 \mathrm{MXN}$. The groups that presented more absences and therefore generated more costs were those corresponding to the month of October, the category of professional nursing, the night shift, in relation to medical disability in January, the unjustified absence presented the highest peak in the month of October. Conclusion: absenteeism produces high economic costs, which stand out in certain groups, by identifying them it is possible to monitor these, and develop strategies that intervene in the causes that originate it, and thus make timely and pertinent decisions.
\end{abstract}

Keywords: absenteeism; Nursing administration research; Nursing Personnel; costs and cost analysis.

${ }^{1}$ Universidad Autónoma de San Luis Potosí, México.

Orcid ID: 0000-0002-3633-5078

2Universidad Autónoma de San Luis Potosí, Salinas de Hidalgo, México.

Orcid ID: 0000-0001-9575-3248

${ }^{3}$ Universidad Autónoma de San Luis Potosí, México.

Orcid ID: 0000-0003-1099-8719

${ }^{4}$ Universidad Autónoma de San Luis Potosí, México

Orcid ID: 0000-0003-4428-2970 


\section{INTRODUCCIÓN}

La Organización Mundial de la Salud (OMS, 2009) refiere que el ausentismo laboral es un fenómeno que se presenta tanto en el personal que realiza tareas administrativas como en el que realiza actividades manuales. De manera que el ausentismo afecta negativamente al desenvolvimiento normal de una organización, pues reduce su productividad e incrementa los costos (Burgos et al., 2018). En la literatura se ha destacado que en los hospitales se presenta un mayor número de ausencias del personal de enfermería quienes son el personal más numeroso de la plantilla, trabaja los 365 días del año y las 24 horas del día, para lo cual se distribuye en jornadas o turnos para proporcionar continuidad en el cuidado. Entre las causas de ausencia se destacan la presión laboral y el estrés, los cuales ocasionan pérdidas de días laborales (Baydoun et al., 2016; Mudaly y Nkosi, 2015).

Por esta razón el ausentismo en los profesionales de enfermería es un fenómeno complejo y multicausal, que afecta a la institución por los costos derivados, al personal de la institución que debe cubrir la ausencia, así como la calidad de atención de los usuarios (Velásquez et al., 2019). Con respecto a las repercusiones al cuidado del paciente Castle y Ferguson (2015) encontraron que los altos niveles de ausentismo están asociados con un bajo rendimiento en los indicadores de calidad, además, incrementa los costos en la atención. Por otro lado, hay una serie de dificultades relacionadas con el ausentismo que incluyen costos directos e indirectos, entre los directos se incluye el pago del personal de reemplazo. Es por lo que, investigaciones como la de Vidal et al. (2017) afirman que el ausentismo laboral genera costos que pueden ser cuantificables por el valor económico de un día no laborado.

Las ausencias en enfermería generan una preocupación creciente en la Administración ya que tienen el desafío de asegurar la prolongación de los cuidados, pues una ausencia no programada puede ocasionar afectaciones en la dinámica de los servicios, afectando a los miembros del equipo y al entorno de trabajo (Ticharwa et al., 2019). Conjuntamente contribuyen a la inestabilidad del personal, generan insatisfacción laboral, sobrecarga de trabajo en los profesionales compromete la calidad de la atención y aumenta los costos de una organización originando implicaciones financieras en todos los niveles, Además, el impacto económico que sufre la institución no se deriva únicamente de las tareas específicas que se dejan de desempeñar, sino genera costos asociados al entrenamiento de nuevos trabajadores y pago de horas extras (Baydoun et al., 2016; Heba et al.,2021; Kanwal et al., 2017).

El ausentismo laboral es un fenómeno multifactorial ocasiona que tiempo y recursos se dilapiden. Al respecto Huber (2018) afirma que se ha reportado en Estados Unidos de América, que algunas compañías pierden hasta 3600 USD al año por cada trabajador que se ausenta. Rispel y Moorman (2015) encontraron que en Sudáfrica un Hospital pagó 5.55 millones USD anuales por las suplencias en enfermería. Mientras que otro hospital de este mismo país reportó un gasto adicional de 1.49 millones USD anuales por el mismo concepto. Maltezou et al., (2021) estudiaron los costos asociados al COVID-19 y encontraron que el ausentismo fue un impulsor predominante de gastos para las instituciones sanitarias entre ellas salarios perdidos. El ausentismo afecta cada vez más a organizaciones y empleadores, esta situación debe considerarse a la luz de las tendencias de reducción de costos que sustentan la nueva administración de la asistencia sanitaria, bajo un enfoque de eficiencia financiera y del uso del tiempo del personal (Ticharwa et al., 2019).

El modelo económico del ausentismo laboral propuesto por Kaiser (1998), considera que el comportamiento del ausentismo obedece a la interacción de dos fuerzas: las motivaciones individuales del trabajador para ausentarse y la tolerancia de los empleadores respecto al número de ausencias. Los trabajadores eligen la cantidad de ausencias que maximizan sus utilidades. Por su parte, los administradores pueden tolerar un cierto nivel de ausencia de los trabajadores, ya que al igual que ellos, efectúan el cálculo de los beneficios pueden estar dispuestos a tolerar altas tasas de ausencia, ofreciendo salarios más bajos. Al abordar el ausentismo en países de Latinoamérica, Tatamuez (2018) considera que se debe actuar de manera integral, atendiendo la calidad de vida, la salud mental del trabajador, la competitividad y la economía de la organización, con el propósito de intervenir con efectividad.

En México la literatura sobre el costo del ausentismo en enfermería es escasa, a nivel local no se identificó, por lo que la presente 
investigación aborda la problemática del ausentismo laboral atribuible a falta injustificada e incapacidad médica, situación que genera fuertes costos económicos y organizacionales que afectan la productividad de la Institución de Salud. También se determinó el costo del ausentismo, lo cual permitirá planificar estrategias que contribuyan a tomar decisiones para su contención en el personal de enfermería.

\section{METODOLOGÍA}

Estudiodeenfoquecuantitativodetipodescriptivo, retrospectivo, transversal, con registros de asistencia del personal de enfermería de un Hospital Público de Segundo Nivel, de San Luis Potosí, México. La investigación fue revisada y aprobada por el comité de ética en investigación, y por el Comité de Investigación del Hospital en estudio. Para determinar el ausentismo laboral se analizan los registros diarios de asistencia del año 2019. Las categorías del personal de enfermería se agrupan atendiendo a los perfiles establecidos por la Secretaría de Salud a través de la Comisión Permanente de Enfermería (CPE) (Secretaria de Salud, 2006) a saber: (a) enfermeras no profesionales que incluye a las Auxiliares y Técnicas en Enfermería; (b) Enfermeras Profesionales integrado por Licenciadas en Enfermería y Enfermeras Generales y; (c) Enfermeras con Posgrado, a las enfermeras con Maestría o Especialidad.

Para determinar los índices se siguieron las recomendaciones de Taylor (1989) para la Organización Internacional del Trabajo (OIT), y las de Molinera (2006) quienes describen las medidas más habituales en los estudios y análisis de ausentismo, de esta manera se mide la actuación del ausentismo a lo largo del año. Los indicadores son datos en series temporales, que reflejan y registran cambios a través de un número significativo de dimensiones relevantes su uso posee estabilidad en las medidas a lo largo del tiempo y son consistentes a través de distintas situaciones (Molinera, 2006). En este sentido es que se analizó la Tasa global de ausentismo o Índice de ausentismo absoluto, el cual, indica el porcentaje de los días de trabajo perdidos en relación con el total de días de trabajo previstos o programados. Este cálculo, muestra como la institución se ve afectada por un incremento en sus costos por la inactividad compensada, causado por los días no trabajados y pagados; situación que afecta de manera directa e indirecta las relaciones laborales entre los trabajadores. Por su parte, el Índice de gravedad o severidad es la duración promedio de la ausencia por persona (Molinera 2006; Taylor 1989). Para estos cálculos se utilizan las abreviaturas de Inasistencia por falta injustificada (IFI), e Inasistencia por incapacidad médica (IIC)

Tasa global de ausentismo laboral

$=$ №. de turnos ausentes por total de inasistencias (IFI y $\| \mathrm{C}$ ) en el periodo Plantilla media para el periodo

Índice de gravedad (IG)

$=\underline{\text { No }}$. de turnos totales perdidos $(\mathrm{IFI}$ y $\| \mathrm{C}$ ) en el periodo No. de personas que tuvieron ausentismo (IFI y IIC) en el periodo

El universo fueron los registros de asistencia, la población que incluye 3965 registros de ausentismo del personal de enfermería durante el año 2019. Se estudió el total de población que reunió los criterios de inclusión. Se incluyen los registros del personal de enfermería que presentó ausentismo por incapacidad, o falta injustificada. Se excluyeron los registros del personal que no sea parte de la actividad de Enfermería, así como el ausentismo justificado por derechos laborales. El costo del ausentismo se calcula a partir del número de horas laborales y número de días de ausentismo, para así estimar el costo por turno o jornada laboral.

Se diseña un instrumento de recolección de datos exprofeso, que consta de tres partes. La primera contiene características demográficas: sexo, edad, formación, rango de edad. La segunda las laborales: categoría, antigüedad, servicios y áreas. En la tercera se registran el número de faltas por persona. Para estimar el costo económico del ausentismo laboral se hizo en una base de datos en Excel, se registraron los costos de salario integrado, costo por turno, el número de turnos perdidos, número de días de ausentismo, área de trabajo, jornada laboral, y sueldo bruto mensual, con la siguiente formula: costo total ausentismo (CTA), turno total perdido en el periodo (TTPEP) y costo promedio estimado por turno (CPET).

Costo total del ausentismo

$\mathrm{CTA}=\mathrm{TTPEP}{ }^{*} \mathrm{CPET}$ 


\section{RESULTADOS Y DISCUSIÓN}

Durante el año 2019, se encontró que 507 elementos del personal de enfermería que representan el $79 \%$ de la plantilla total, presentaron ausentismo laboral de las cuales se determinaron 3965 turnos de ausencias, de los cuales $69 \%(2751)$ corresponde a faltas injustificadas, y $31 \%(1214)$ a incapacidades médicas. De acuerdo al rango de edad predominante fue de 45-54 años con una media de 45.40 años y una mediana de 46 años, este resultado es similar al encontrado por (Castillo et al., 2016) quienes observaron que los individuos de más de 45 años presentaron mayor ausentismo. Kunrath et al., (2021) encontraron que la edad media de los enfermeros ausentistas fue de 45,05 \pm 9,7 años. Se observa que los trabajadores de mayor edad presentan más ausencias, vale decir que los trabajadores de 50 años o más aumentan en un $85 \%$ (Vidal et al., 2017).

De acuerdo al turno la incapacidad médica se presentó en mayor porcentaje en el turno vespertino y la falta injustificada en el matutino y nocturno, en ambos tipos de ausentismo se presentó con más frecuencia en el personal móvil, es decir el personal que no tiene un servicio fijo y se encuentra realizando sus turnos según la disposición de Jefatura de Enfermería, en segundo lugar, el área de Urgencias, y el tercer lugar Quirófano. En lo referente a faltas injustificadas el primer lugar fue el área móvil, en segundo lugar, Quirófano y en tercer lugar Urgencias. Ticharwa et al., (2019) mencionan que trasladar a las enfermeras a cubrir la escasez en otros servicios causa malestar psicológico y contribuye al ausentismo, dicha situación se observa en el hospital de estudio y puede ser un detonante del propio ausentismo.

Por otro lado, Kunrath et al., (2021) refieren que el servicio de urgencias es un entorno donde a menudo se experimenta sufrimiento y muerte, lo que ocasiona inestabilidad, un clima de expectativas y ansiedad en el equipo de atención lo que podría conllevar a ausentismo. Por categoría, predominan las ausencias del personal de Enfermería Profesional 76.5\% (3029), seguido de Enfermería No Profesional $13 \%$ (518), y en tercer lugar Enfermería con Posgrado 10.5\% (418).

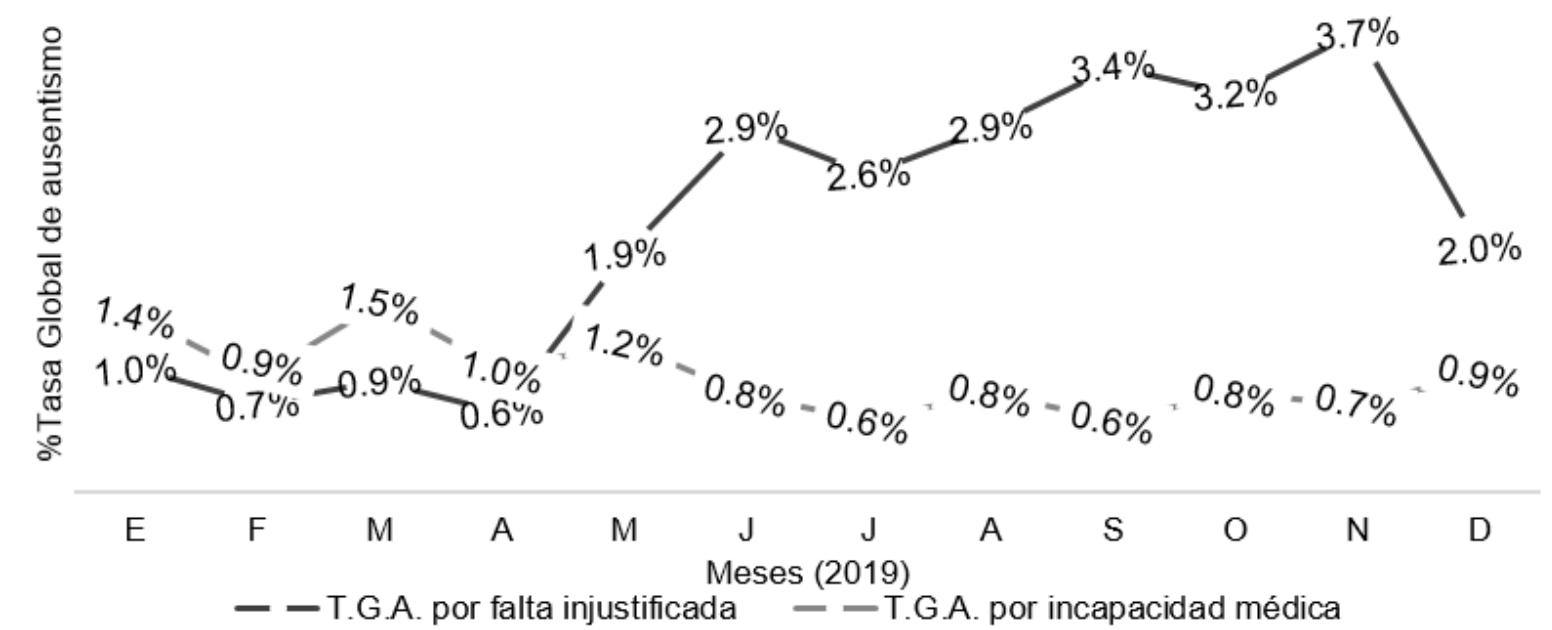

Figura 1

Tasa Global de Ausentismo (TGA) mensual para el año 2019

En relación a la Tasa Global de Ausentismo (TGA) en la figura 1 se observa que el mes más bajo de la tasa anual por falta injustificada fue en el mes de abril, a diferencia del mes de noviembre que fue el más alto, por otro lado, la tasa de incapacidad tiene tendencia baja encontramos pequeñas variaciones a lo largo del año siendo sus picos más altos en el mes de marzo y diciembre. Es importante considerar que el ausentismo justificado no se encuentra contemplado en la figura. La tasa normal de ausentismo se sitúa entre el $3,5 \%$ y el $4,5 \%$ en los trabajadores que realizan tareas administrativas y 
entre el $5,5 \%$ y el $6,5 \%$ en los trabajadores con profesiones manuales (OMS, 2009). Cabe destacar que en el estudio que presentan los autores no se considera el ausentismo justificado por legalidad institucional, lo cual influye en los resultados reportados. Dichos niveles son similares a los reportados por Velásquez et al., (2019) quienes hallaron que la Tasa Global de Ausentismo fue de $5.7 \%$ en un hospital peruano.

En la figura 2 encontramos el índice de gravedad (IG) en el 2019, indica que en promedio las faltas de las personas duraron dos días. Tomando en cuenta la figura 2, se observa que el IG en incapacidad médica fue más alto en el mes de enero, mientras que el índice de gravedad por falta injustificada fue más alto en los meses de septiembre- noviembre. El IG representa la duración promedio en días de las ausencias que fueron presentadas en la población laboral analizada (Taylor, 1989). Las cifras encontradas son menores a las presentadas por Velásquez et al., (2019) quienes encontraron que la duración promedio de sus ausencias fue de 6 días. Maltezou et al., (2021) enfatiza que el ausentismo es un problema grave que se atribuye en parte a su prolongada duración y su potencial impacto en los costos.

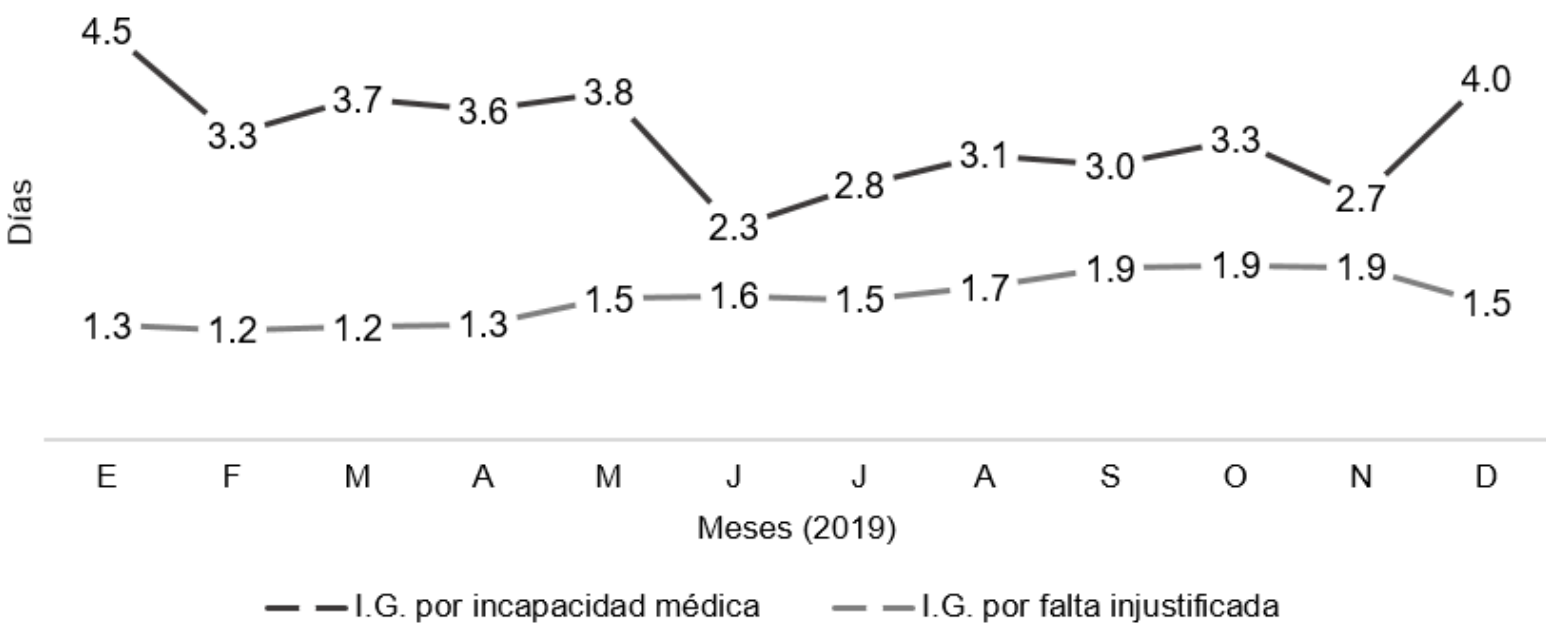

Figura 2

Índice de gravedad (IG) mensual para el año 2019

En función de la determinación del costo total del año 2019 en la figura 3, se observa que el costo del ausentismo se incrementó notablemente en los meses de mayo a diciembre 2019 siendo el mes de octubre el más costoso representando el $11.5 \%$ del total, mientras que el mes que menos costo ocasionó fue el mes de febrero que representa el $4 \%$ del costo total, siendo el costo anual estimado de $\$ 6676134.62$ pesos mexicanos (MXN), en promedio el costo diario estaría alrededor de $\$ 18$ 291 (MXN). Rispel y Moorman (2015) encontraron que en Sudáfrica un Hospital gastó diariamente $\$ 15,205$ USD por las suplencias en enfermería. Mientras que otro hospital de este mismo país gasto $\$ 4,802$ USD diarios aproximadamente, costos que no estaban previstos por la institución. Kanwal et al., (2017) respaldan la idea de que el ausentismo es muy oneroso tanto para los empleados como para el equipo directivo, ya que el trabajador que se ausenta debe remplazarse para que su trabajo sea realizado y de esta manera brindar una atención oportuna al paciente. 


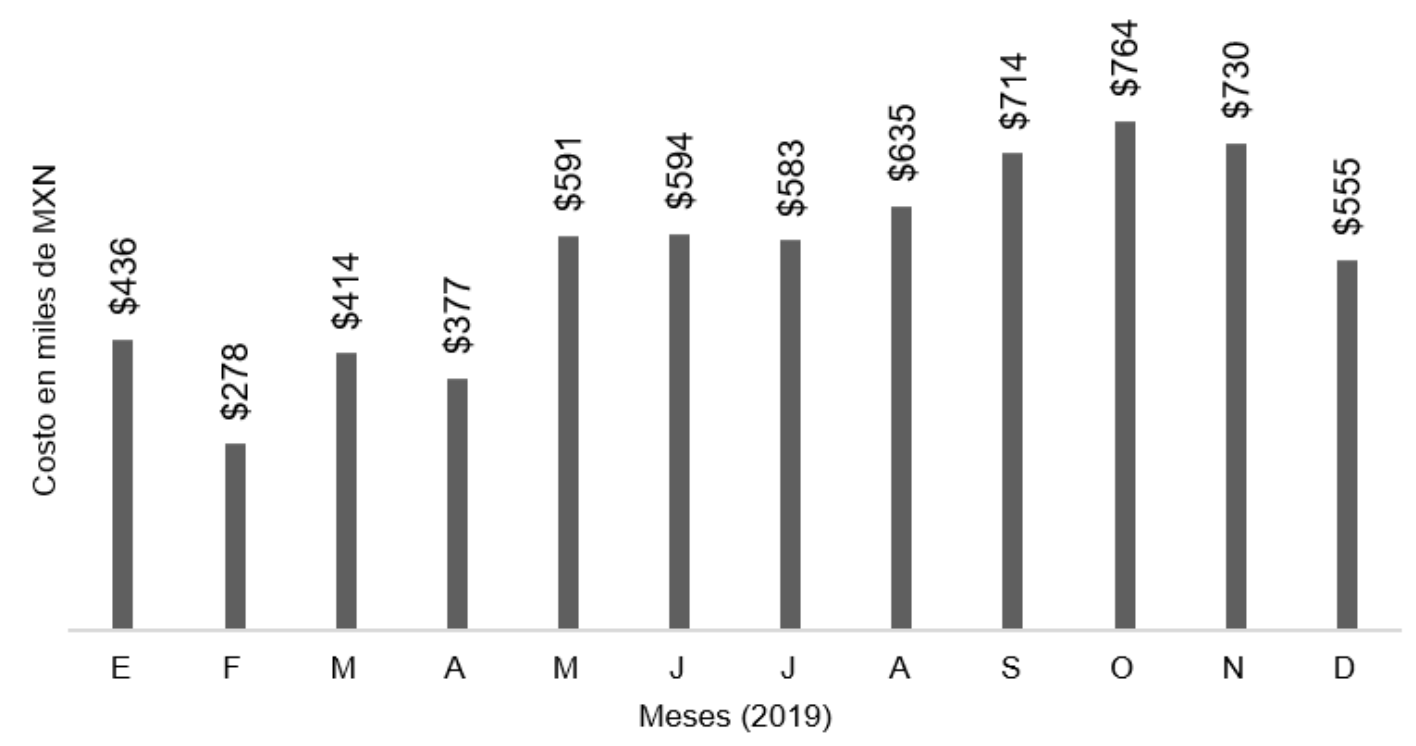

Figura 3

Trayectoria mensual del Costo del Ausentismo laboral en Enfermería en el año 2019

En la figura 4, destaca que la categoría Enfermera Profesional fue la que mayor costo total genera con una participación del $80 \%$ del total. La formación académica que prepondera en este estudio fue la de Licenciatura en Enfermería. Al relacionar el ausentismo con diversas profesiones del área sanitaria, Castillo et al., (2016) reportan que la función de enfermería es la que presenta mayor ausentismo, encontrando una relación estadísticamente significativa de la profesión con el ausentismo. Dichos resultados discrepan con los resultados de Castle y Ferguson (2015) quienes reportan que las auxiliares de enfermería tienen altas tasas de ausentismo.

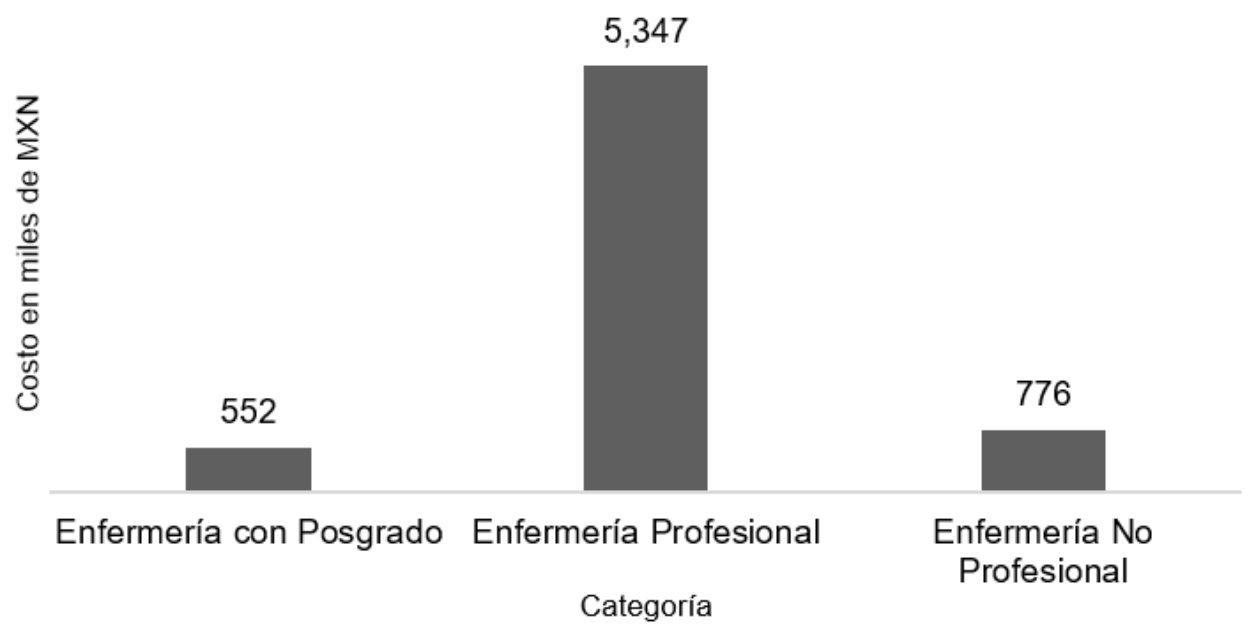

Figura 4

Costo anual (2019) del Ausentismo laboral, por categoría de Enfermería

En la figura 5 se hace evidente que las inasistencias a los turnos nocturnos fueron las que más generaron costos comparado con el total: nocturno $48 \%$, vespertino $26 \%$ y matutino $26 \%$. En un 
análisis por meses, en miles de MXN, se observa que el mes de septiembre el que más genera en el turno nocturno representando un $5.4 \%$ (362) del costo total anual. El turno matutino que más costo presenta fue el del mes de octubre con $3.7 \%(246)$ del costo total anual. El turno vespertino que más costo ocasiona fue el del mes de octubre $2.9 \%$ (192) del costo total. Lo encontrado concuerda con Ticharwa (2019) quienes consideran que el laborar en turnos nocturnos es un factor que contribuye al ausentismo, dado que los trabajadores nocturnos son más propensos a la fatiga y condiciones crónicas; por lo tanto, tienden a estar ausentes con mayor frecuencia. Mudaly (2015) menciona que las ausencias se dan debido a la tensión laboral, las largas horas de trabajo, además, las malas condiciones de trabajo; afectan la escasez del personal, ya que el personal restante se agota aún más y se ausenta del trabajo. Kunrath (2021) afirma que las enfermeras que más se ausentaron laboraban en el turno de noche, seguidas del turno de mañana.

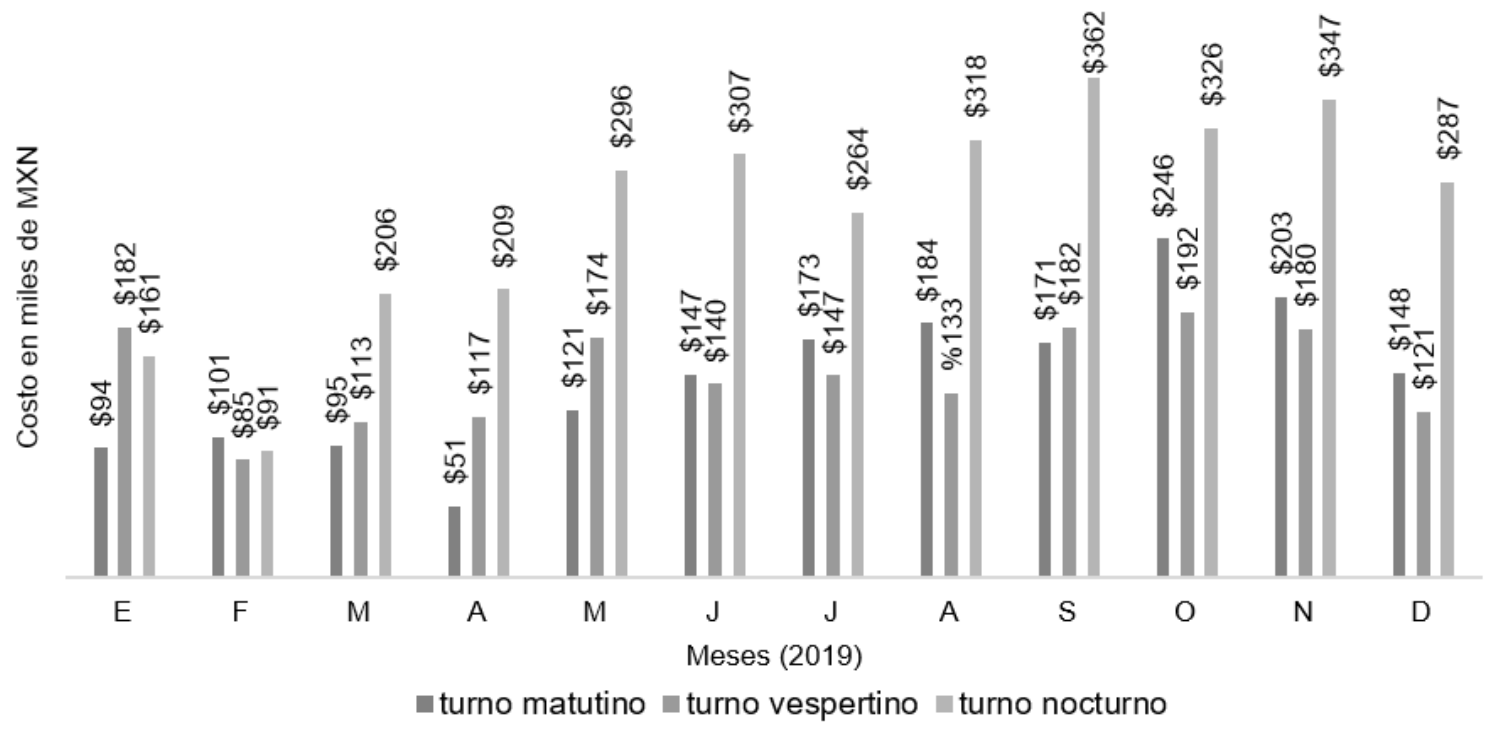

Figura 5

Costo mensual del de Ausentismo laboral de Enfermería por turnos, 2019

En la figura 6 se muestra que los costos mensuales por incapacidad médica fueron mayores en el mes de enero, con un valor de $3.7 \%$ estimando un costo de $247 \mathrm{MXN}$ mientras que el costo por falta injustificada aumentó marcadamente desde mayo con un valor de $5.3 \%$ estimando un costo de 351 MXN teniendo el pico más alto en el mes de octubre representando $9.3 \%$ con un valor estimado de $627 \mathrm{MXN}$.

Las faltas injustificadas se pueden presentar debido a afecciones en las áreas de la vida laboral, estas ausencias podrían darse debido a impactos en la salud psicológica de los trabajadores, insatisfacción laboral, agotamiento, este problema se vincula directamente con perjuicios y costos para la institución (Heba et al., 2021). Existen diversas causas por las que los trabajadores presentan faltas injustificadas las más frecuentes son por enfermedad intempestiva. Algunas veces las incapacidades médicas no responden a problemas de salud, sino al deseo del trabajador de no presentarse a laborar, desestimando el costo que ello representa para el hospital, porque tiene que disponer de trabajadores extra a quienes deberá capacitar y remunerar por el trabajo adicional (Velásquez et al., 2019). En el hospital de estudio no se contrata personal extra, ya que el personal denominado móvil es un personal fijo cuya función es suplir las faltas, es decir, no se contrata personal eventual, sino que generan un costo permanente. Además, como ya se expresó anteriormente, este es el grupo que más ausencias presenta a lo largo del año. 


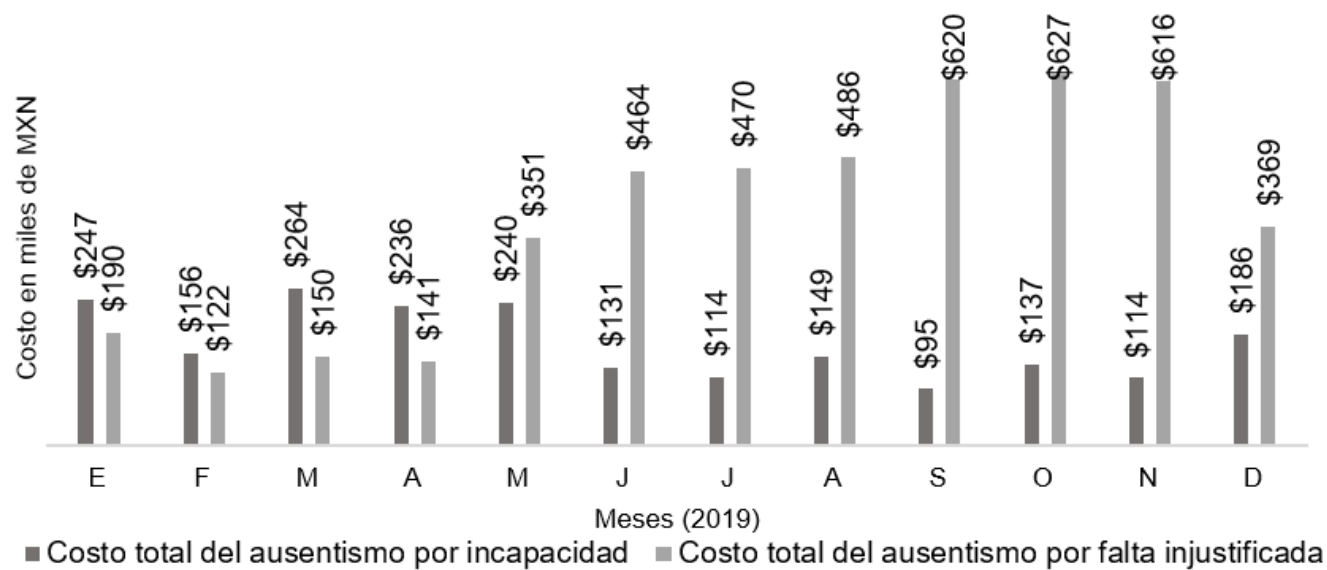

Figura 6

Costo mensual del de Ausentismo laboral de Enfermería por tipo de ausentismo, 2019

Otras causas que podría desencadenar las incapacidades en el equipo de Enfermería puede deberse a la exigencia en su labor, el gran esfuerzo físico, esto sumado al envejecimiento fisiológico, reduce la capacidad de producir respuestas adecuadas a los factores estresantes internos y externos, provoca una predisposición a enfermedades, trastornos cardíacos, respiratorios, resistencia muscular, pérdida de fuerza, y una disminución en los componentes del equilibrio y coordinación, estas características, sumadas a las responsabilidades de su vida social cotidiana, llevan al individuo a situaciones de estrés y enfermedad, las cuales generan patologías que se pueden volver crónicas y requerirán de atención (Kunrath et al., 2021; Tatamuez et al., 2018).

En la actualidad se debe considerar el impacto general de la pandemia de COVID-19 en el ausentismo laboral relacionado con la salud, Groenewold et al., (2020) encontraron que el ausentismo fue significativamente mayor de lo esperado entre varios grupos ocupacionales que se involucran inherentemente al contacto cercano prolongado con pacientes, considerados esenciales y críticos. Es probable que los trabajadores de estos grupos ocupacionales hayan tenido que seguir estando físicamente presentes en sus lugares de trabajo y no pudieron evitar la exposición, también que los trabajadores en estas ocupaciones esenciales tengan un mayor riesgo de exposición ocupacional al SARS-CoV-2 y, por ende, estén más propensos a enfermar y ausentarse. Maltezou et al., (2021) realizó un análisis de los costos asociados al
COVID-19, entre los costos indirectos enfatizó que el ausentismo, es el principal impulsor de los costos totales, representó un $80,4 \%$ de todos los gastos.

De acuerdo con el trabajo presentado y a los resultados obtenidos, se pueden plantear las siguientes conclusiones principales:

A pesar de no haber considerado las ausencias por legalidad institucional, el ausentismo del personal de enfermería produce altos costos adicionales, que se incrementó notablemente en los meses de mayo a diciembre 2019, siendo el mes de octubre el más costoso mientras que el mes que menos costo ocasiono fue el mes de febrero se determinó que el costo económico estimado anual, fue de $\$ 6,676$ mil MXN. Recursos que pudieran programarse para aumentar la calidad y cantidad de pacientes atendidos. En cuanto a la categoría que genera mayores costos por ausentismo es la enfermera profesional en comparación a la enfermera no profesional ( $\$ 5,347 \mathrm{mil} M X N$ vs $\$ 553$ mil MXN). Se hace evidente que los turnos nocturnos fueron los que más generaron costos, siendo el mes de septiembre el que más costo generó en el turno nocturno \$ 362 mil MXN. En relación a los costos perdidos por incapacidad médica fueron mayores en el mes de enero, con un valor estimado de $\$ 247$ mil MXN mientras que el costo por falta injustificada aumentó marcadamente de mayo a diciembre con un valor estimado de $\$ 351$ mil MXN teniendo el punto más alto en el mes de octubre con un valor estimado de \$ 627 mil MXN. Esto implica la 
necesidad de generar evidencia de la existencia de factores sociodemográficos en el personal que condicionen el ausentismo para poder actuar en consecuencia.

El hospital de estudio posee un grupo de personal que se dedica a laborar según disposición de la Jefatura de Enfermería cubriendo las ausencias en diferentes servicios, el presente estudio revela que este personal fue el que más se ausentó durante el 2019. Se ponen de manifiesto ineficiencias en el gasto programado, los procesos de administración y gestión en la organización. El tener un registro ordenado del ausentismo laboral, ayudará a evaluar tendencias, y elaborar comparaciones, además, con ello se podría llevar un seguimiento individualizado para generar planes para impedir la recidiva. Se recomienda que se realice un programa estructurado que incluya actividades de prevención primaria, capacitación, diagnóstico, control de factores de riesgo psicolaborales, intervenciones ergonómicas, intervenciones de control de peso y anti sedentarismo, para favorecer de manera importante la salud de los trabajadores, la reducción del ausentismo en la institución, así como sus costos.

\section{Declaración de financiamiento y de conflictos de interés}

El estudio fue financiado por los autores, quienes declaran no tener algún tipo de conflicto de interés en la investigación realizada

\section{Correspondencia}

\section{Paulina Tipantuña-Malte \\ Correo electrónico: \\ raqueltipantuna@gmail.com}

Armando Sánchez -Macias
Correo electrónico:
armando.sanchez@uaslp.mx

\section{Sofia Chevarria -Rivera}

Correo electrónico: sofia@uaslp.mx

\section{Rosa Andrade Cepeda \\ Correo electrónico: randradebc@hotmail.com}

\section{REFERENCIAS}

Baydoun, M., Dumit N., Daouk L., (2016) What do nurse managers say about nurses' sickness absenteeism? A new perspective, https://doi. org/10.1111/jonm.12277, J. Nurs. Manag., 24(1), 97-104

Burgos, L., Faggioni, A. y Pérez, J., (2018) Gestión de talento humano en relación al ausentismo $y$ rotación de personal en las empresas constructoras de la ciudad de Guayaquil, Observatorio de la Economía Latinoamericana

Castillo, M., Castro C., otros 4 autores. (2016) Ausentismo laboral y factores de riesgo cardiovascular en empleados públicos hospitalarios, Acta Bioquim. Clin. Latinoam., ISSN 0325-2957, 50(1), 37-44

Castle, N., Ferguson J., (2015) Influence of nurse aide absenteeism on nursing home quality, https://doi. org/10.1093/geront/gnt167, Gerontologist., 55(4), 605-615

Groenewold, M., Burrer, S., y otros 4 autores, (2020) Increases in Health-Related Workplace Absenteeism Among Workers in Essential Critical Infrastructure Occupations During the COVID-19 Pandemic United States, March-April 2020, https:// doi.org/https://dx.doi.org/10.15585\%2Fmmwr. mm6927a1, MMWR Morb. Mortal. Wkly. Reps., 69(27), 853-858

Heba, M., Fatma A., y Soad A., (2021) The influence of Work life Fit Areas and Work life Interference on Nurses' Absenteeism and Performance, J. Nurs. Sci, ISSN: 2320-1959, 10(2), 25-35

Huber, D., (2018) Gestión de los cuidados Enfermeros y Liderazgo. $6^{a}$ edición., Barcelona, España, Elsevier

Kaiser, C. (1998) What do we know about employee absence behavior? An interdisciplinary interpretation, https://doi.org/10.1016/S10535357(99)80078-X, J. Socio. Econ., 27(1),79-96

Kanwal, N., Riaz G., y otros 2 autores, (2017) Identify the Causes of Absenteeism in Nurses Mayo Hospital Lahore Pakistan, https://doi.org/10.3126/ ijssm.v4i2.17171, IJSSM., 4 (2), 110-114

Kunrath, G., Santarem M., y otros 4 autores. (2021) Predictors associated with absenteeismdisease among Nursing professionals working in an emergency hospital service, https://doi. org/10.1590/1983-1447.2021.20190433, Rev Gauch Enferm, (42), 1-8 
Molinera, J., (2006) Absentismo laboral: Causas, Control y análisis. Nuevas formas. Técnicas para su reducción. $2^{a}$ ed., Madrid, España, CONFEMETAL

Maltezou, H., Giannouchos T., y otros 8 autores (2021) Costs associated with COVID-19 in healthcare personnel in Greece: a cost-of-illness analysis, Available from: https://doi.org/10.1016/j. jhin.2021.04.018, J Hosp Infect.,30 (40), 1-8

Mudaly, P., Nkosi Z., (2015) Factors influencing nurse absenteeism in a general hospital in Durban South Africa, https://doi.org/10.1111/jonm.12189, J. Nurs. Manag., 23(5), 623-631

Rispel, L., Moorman J., (2015) The indirect costs of agency nurses in South Africa: A case study in two public sector hospitals, https://doi.org/10.3402/ gha.v8.26494, J. Glob. Health., 8(1),1-9

Tatamuez, R., Domínguez., A, Matabanchoy S., (2018) Revisión sistemática: Factores asociados al ausentismo laboral en países de América Latina, http://dx.doi.org/10.22267/rus.19201.143 Univ. y Salud., 21(1),100-12
Taylor, P., (1989) Enciclopedia de Salud y Seguridad en el Trabajo. Ministerio de Trabajo y Seguridad Industrial, España, Organización Internacional del Trabajo

Ticharwa, M., Cope V., Murray M., (2019) Nurse absenteeism: An analysis of trends and perceptions of nurse unit managers, https:// doi.org/10.1111/jonm.12654, J. Nurs. Manag., 27(1),109-116

Vidal, C., Palavecino I., y otros 3 autores, (2017) Calidad de Vida del Personal de Salud y su Relación con el Ausentismo. http://dx.doi. org/10.4067/S0718-24492017000300188, Cienc. Trab., 60(1),188-193

Velásquez, R., Loli R., Sandoval M., (2019) Factores asociados al ausentismo laboral de enfermeras Factors Associated to Work Absenteeism in Nurses, Rev. Cubana. Enfermer., ISSN: 08640319,35(4), 1-13

World Health Organization (WHO)., (2009) The Global Occupational Health Network, GOHNET., 15(1)

Recibido: 07/07/2021

Aceptado: 15/10/2021 